\title{
Scalable Pitch-Constrained Neural Processing Unit for 3D Integration with Event-Based Imagers
}

\author{
Maxence BOUVIER \\ Univ. Grenoble Alpes \\ CEA, List \\ F-38000 Grenoble, France \\ maxence.bouvier@cea.fr
}

\author{
Alexandre VALENTIAN \\ Univ. Grenoble Alpes \\ CEA, List \\ F-38000 Grenoble, France \\ alexandre.valentian@cea.fr
}

\author{
Gilles SICARD \\ Univ. Grenoble Alpes \\ CEA, Leti \\ F-38000 Grenoble, France \\ gilles.sicard@cea.fr
}

\begin{abstract}
Event-based imagers are bio-inspired sensors presenting intrinsic High Dynamic Range and High Acquisition Speed properties. However, noisy pixels and asynchronous readout result in poor energyefficiency and excessively large output data rates.

In this work, we use Convolutional Spiking Neural Network filters to compensate these drawbacks and reduce output bandwidth by 10x.

We designed a neuromorphic core as a distributable block that benefits from 3D integration technology with direct and parallel access to 32x32 pixels, enabling reduced frequency operation. Post-layout simulations depict a peak energy efficiency with $2.83 \mathrm{pJ}$ per Synaptic Operation (equivalent to $0.093 \mathrm{fJ} / \mathrm{event} / \mathrm{pix}$ ) at the nominal literature input event rate.
\end{abstract}

Index Terms-Spiking Neural Networks, Neuromorphic, 3D IC Technology, Near-Sensor Computing, Event-Based Vision

\section{INTRODUCTION}

We observe an increasing number of applications where being able to reliably distinguish movement or objects is necessary, e.g. autonomous machines like drones or taxis, or mixed reality. Under certain conditions, to acquire images whose quality is high enough to guarantee object detection or movement evaluation may require the use of High Dynamic Range (HDR) and High Acquisition Speed (HAS) cameras. Moreover, most of these applications concern embedded devices or machines, which generally require low power components and reduced form factor. Traditional CMOS imagers deliver exhaustive and spatio-temporally redundant data, and usually do not combine HDR and HAS properties without post-treatment.

Thus, we focus on Event-Based (EB) imagers [1], [2], which have successfully been used for movement evaluation and autonomous navigation [3]. Because of the bio-inspired behavior of EB image sensors, they naturally combine HDR, HAS, and event-based data acquisition. The latter means that the sensor does not deliver any data (noise excepted) when nothing occurs - i.e. when the sensor and the scene are motionless. No redundant data are generated nor treated, thus minimizing power consumption. However, EB imagers come with several drawbacks. They are noisy and hardly scalable at HD resolutions because of the required output bandwidth.

As a solution, this work presents a neural processing unit evaluating a Convolutional Spiking Neural Network (CSNN) conceived to be easily distributed behind an EB pixel grid by employing 3D IC technologies [4], [5]. Analogously to biological neurons of the striate cortex [6], the CSNN evaluated by the neuromorphic core detects oriented edges in the raw input EB data stream. It reduces data throughput by up to an order of magnitude and filters out noise; thus minimizing several drawbacks of raw EB data streams, while conserving spatial and temporal information. Such construct would be a first step in the realization of a complete bio-inspired vision system, and the initial motivation for this work was to verify that CSNN algorithm could be implemented near-sensor.

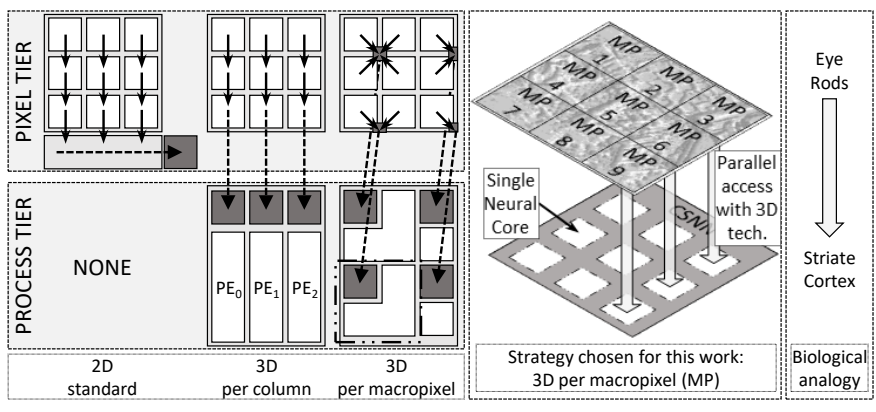

Fig. 1: Left: illustration of different 3D readout possibilities. Right: chosen strategy and analogous biological system.

Our neural core evaluates 256 convolutional spiking neurons and consumes only $2.86 \mathrm{pJ}$ per Synaptic Operation (SOP). It takes advantage of the envisaged 3D parallel interconnection with a block of $32 \times 32$ pixels by integrating the pixel readout module which encodes the spikes' address in a custom format that allows for optimized synaptic mapping information - i.e. weights and associated neuron addresses - storage requiring only 300 bits. The timing constraints on the arbiter is also drastically reduced. Moreover, the neural core can be tiled to manage high resolution sensors without inducing overhead and the readout. The allowed surface is constrained by the pixels above the core, whose pitch is set at $5 \mu \mathrm{m}$ targeting the state of the art 720p EB imager [7] characteristics, which results in a core surface of only $0.026 \mathrm{~mm}^{2}$. To the best of our knowledge, it is the first SNN hardware accelerator realized targeting a near-sensor implementation.

Related works are introduced section II, and algorithm to hardware optimization efforts are analyzed section III. Section IV depicts the circuit architecture, and post-layout results are presented and compared with other neuromorphic cores section V. We finally conclude in section VI.

\section{RELATED WORKS}

\section{A. Event-Based Imagers}

Event-based imagers are bio-inspired sensors based on asynchronous readout of individual pixels. Each pixel emits an event a spike - when a change in the illumination measured overcomes a threshold [1], [2] as would the rods in human eyes [8]. Whereas simultaneously providing HDR, HAS, and EB data acquisition; they are hardly scalable with standard 2D integration because the complexity of the readout system - called arbiter - increases linearly with the number of cells to read [9]. The output bandwidth tends to reach important values, of the order of tens of $\mathrm{Gb} / \mathrm{s}$, not suitable for low power systems. Moreover, the pixels can be very noisy. 


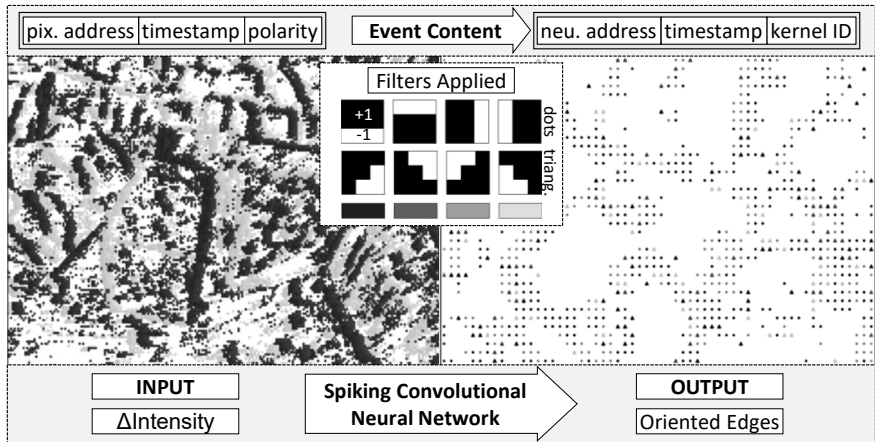

Fig. 2: CSNN results. Left: input data, B\&W dots correspond to -1 $\&+1$ event polarity respectively. Right: corresponding output data, round dots correspond to vertical and horizontal filters, triangles dots correspond to diagonal ones. Input data from [22].

Recent examples of EB sensors trying to solve these problems are [7], [10], [11].

Reference [11] accesses eight pixels simultaneously in order to reduce the timing constraints on the arbiter. Reference [10] filters out faulty pixels and noise by counting and thresholding spikes emitted by group of $2 \times 2$ pixels. Reference [7] exploits 3D IC technology to readout the pixel matrix by row, reducing the arbiter complexity by by 1280 . Moreover, more space is available on the bottom tier thanks to 3D stacking, they thus implemented a filter by Region of Interest to reduce the output bandwidth of the sensor.

\section{B. $3 D$ Integrated Circuit Technology}

Image sensors largely benefit from 3D IC technologies, with for example $\mathrm{Cu}-\mathrm{Cu}$ bonding, for numerous reasons [4], [5]. It provides more space for maximizing the pixel fill factor and allows heterogeneous integration, i.e. conjoint integration of high quality light sensing devices on the pixel tier with efficient readout and complex compute modules on the bottom tier - also called process tier. Several actors have already shown achievements basic 2D imaging technologies alone would not have permitted, for smart or autonomous [12]-[14] and EB [7] imagers. Fig.1 illustrates the 3D readout mechanisms used in these works. The 3D per macropixel (MP) strategy, as employed in [14], enables to homogeneously tile processing cores that have direct parallel access to pixels, straightforwardly permitting distributed processing architectures design on the bottom tier.

\section{Spiking Neural Networks Hardware Implementation}

Hubel and Wiesel have shown that one of the first steps related to visual perception in mammals' brain is edge orientation detection [6]. Similarly, edge orientation discrimination is usually realized by the first layers of CSNNs when trained with bio-inspired methods on EB data [15], [16]. However, simulating SNNs on classical computers is time and power consuming because of the sparse and event-based nature of data and computation flow [17].

Hence, many academic [18]-[20] and industrial [21] actors are working on dedicated hardware for evaluating SNN algorithms. The neural cores presented are usually designed with more [18], [21] or less [19], [20] programmability regarding the final algorithm, with more [18], [21] or less [19], [20] bio-plausibility regarding the neuron mechanisms, and target either shallow [18]-[20] or deep [21] SNN topologies. As a general rule: more programmability or complexity is usually obtained at the expense of energy efficiency, operation speed, or area [17].
TABLE I: CSNN Algorithmic Parameters and Values

\begin{tabular}{|l||c|c|}
\hline Parameter name & Symbol & Value \\
\hline Number of Kernels & $N_{k}$ & 8 \\
\hline RF Width & $W_{R F}$ & 5 pix \\
\hline Threshold Voltage & $V_{\text {th }}$ & 8 \\
\hline Stride & $d_{\text {pix }}$ & 2 \\
\hline Refractory Period & $T_{\text {refrac }}$ & $5 \mathrm{~ms}$ \\
\hline Leakage Type & $f_{\text {leak }}$ & exponential \\
\hline Leakage Time Constant & $\tau$ & $1 / 3^{\text {rd }}$ of $20 \mathrm{~ms}$ \\
\hline
\end{tabular}

Our design mixes several ideas presented by related works. It exploits the benefits that would be brought by combining EB imaging with 3D stacked macropixel readout for implementing a fully hardwired shallow CSNN evaluator. We minimize the flexibility and size of the CSNN implemented for performance matters aiming for embedded operation. The long-term idea of our work is that realizing spatio-temporal edge orientation filtering near-sensor would permit to reduce drawbacks related to EB sensors. We choose to implement the neural core in a data-stream processing perspective to take advantage of the EB nature of data: no computation or data movement is uselessly realized when no input data is available.

\section{Algorithm Hardware Co-Optimization}

\section{A. Convolutional Spiking Neural Network}

A convolutional layer of a CSNN is a mesh of local filters - called neurons - distributed throughout the input frame. Each neuron takes in input a small window of the whole input frame - called Receptive Field (RF). Each spike generated in this window adds a value - called synaptic weight - to the neuron's membranes - called kernel potentials $V_{k_{i}}$. When a kernel potential overcomes the neuron's threshold $V_{t h}$, the neuron emits a spike - it fires. Neurons operating as described are called Integrate and Fire (IF).

CSNNs conserve and exploit the spatio-temporal information contained in the input data. As neurons are regularly distributed throughout the input space, and observe only a small window, spatial information is conserved by labelling output events with the address of the emitting neuron. Temporal information is exploited with two mechanisms. First the leakage, which consists in progressively decreasing the neurons' kernel potentials through time. Contributions of low frequency noisy spikes are thus deleted with time. On the other hand, spikes arriving in quick succession are more likely to make the neuron fire. This denotes that leaky neurons favor temporally correlated input spikes, and consequently limit the impact of noisegenerated spikes. Leakage is the direct equivalent of capacitors discharge in analog electronics. Secondly the refractory period, which limits the maximum output frequency of a neuron by forbidding firing during an amount of time $T_{\text {refrac }}$ after each spike emission. The impact of faulty always-on pixels is thus diminished.

Hence, applying a mono-layer CSNN with Leaky Integrate and Fire (LIF) neurons on a raw stream of events acquired by an EB camera enables to conserve spatial information while reducing noise and event-rates. The output is a new stream of events, each corresponding to a spatio-temporal pattern - called feature or kernel - that fired, as illustrated Fig. 2.

\section{B. From Algorithm to Hardware}

SNN acceleration near-sensor has been discussed but not yet demonstrated [10]. 3D integration facilitates the realization, even more with Convolutional NNs whose locality property is perfectly suited for macropixel arrangement [7], [14] (Fig. 1). However, memory requirements of Spiking NNs, with every neuron states continuously stored, make them area-hungry [18]-[20]. Our objective is thus to permit CSNN evaluation in an area constrained to only the 

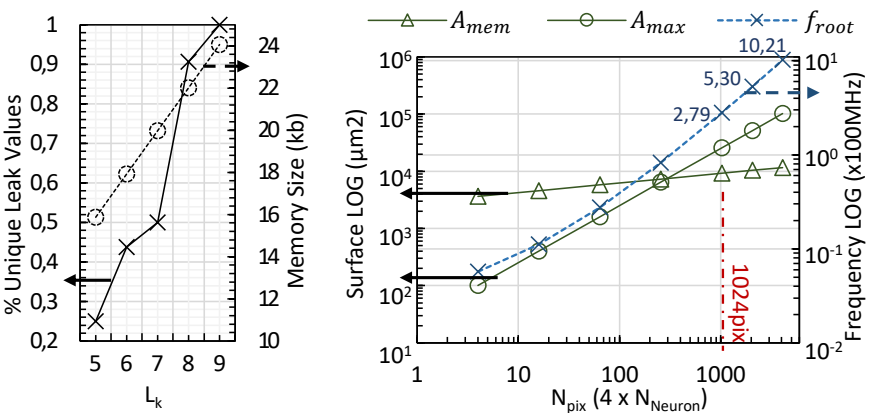

Fig. 3: Design Space Exploration. Left: impact of $L_{k}$ on the LUT precision and $M$. Right: $N_{p i x]}$ trade off between $f_{\text {root }}$ and $A_{m e m}$ requirements. $A_{m e m}$ values are obtained with SRAM cut generation exploration.

area of the pixels above the core in the macropixel construct. For that, we minimize the chip programmability and we apply several standard NNs related hardware optimizations. We also propose a custom method for mapping the pixel grid to the neurons taking advantage of the local 3D interconnections to pixels.

1) Algorithmic Parameters: Apart from the kernel patterns, the neuron threshold value $V_{t h}$, and the refractory period duration $T_{\text {refrac }}$, every algorithmic parameters is fixed and hardwired in the design. We arbitrarily set the distance in pixel between two RF centers - called the stride $d_{p i x}$-, the number of kernels $N_{k}$ per neuron, the RF width $W_{R F}$, and the weight precision. The rest has been set after an exploration that aimed at obtaining a compression ratio $C R=n_{e v_{i n}} \div n_{e v_{\text {out }}}$ of approximately 10 . The resulting parameter values are listed table I.

With the stride set at two, there is only one neuron to evaluate per group of four pixels. $W_{R F}$ is chosen so that neighboring neurons' RFs overlap. The kernel patterns, visible in the inlet Fig. 2, are inspired from oriented edges obtained with Spike Timing Dependent Plasticity (STDP) training, which analogously represent actual neurons of the striate cortex [6], [15]. Finally, because nearbinary weight distribution is sometimes spontaneously obtained by training [16], possible weight values are reduced to -1 or +1 .

2) Standard Optimizations: We deploy a few standard hardware optimization technics for improving the energy and area efficiency of NN accelerators, namely clock-gating, fixed frequency scaling, and approximate computing. Our core behaves as a data-stream accelerator where each module works sequentially on a data, without storing intermediate results (apart from neuron states). Data transits one-way, from input to output, and no time nor energy is lost in a ping-pong between a central processing unit and an external memory. The frequency of each module is adapted to its local data rate; and if a module has no valid data in input, most of its components are clock gated. Approximate computing is realized with reduced bitlengths $L_{X}$ of stored timestamps $\left(L_{T S}\right)$ and kernel potentials $\left(L_{k}\right) . L_{T S}$ is set to represent the full $20 \mathrm{~ms}$ of leak range. Hence, with a LSB corresponding to $25 \mu$ s, 10 bits are sufficient. An additional bit is used as a flag indicating overflow, resulting in $L_{T S}=11$. The bitlength of the kernel potentials $L_{k}$ is set to guarantee high precision exponential leakage. Each time a neuron state is loaded, leak is applied by multiplying every kernel potential with the decrement factor leak $k_{v a l u e}=\exp \left(-\left(t_{\text {curr. }}-t_{\text {in }}\right) / \tau\right)$, where $t_{i n}$ is the timestamp of the last input spike. Leak values are stored in a 64-input Look Up Table (LUT) and the left graph of Fig. 3 depicts the variation of the precision - actually of the number of identical decrement factors stored - of the LUT with $L_{k}$ (left axis).

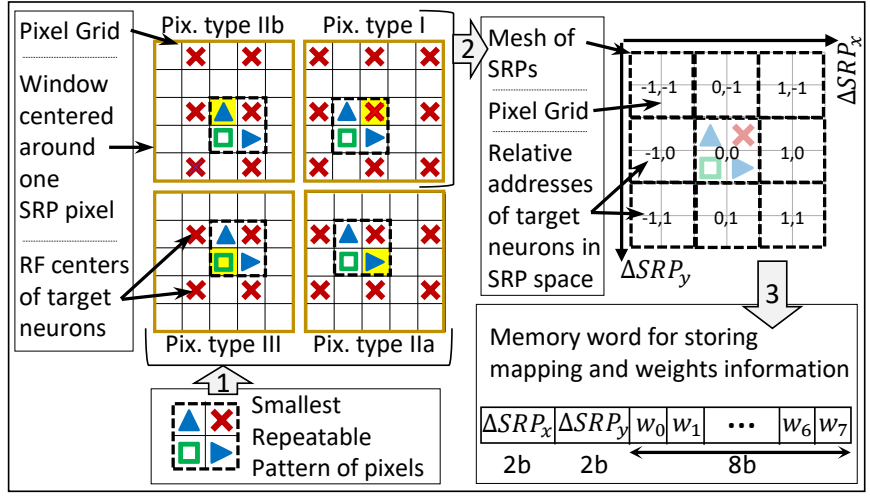

Fig. 4: Smallest Repeatable Pattern definition and associated network mapping memory storage strategy.

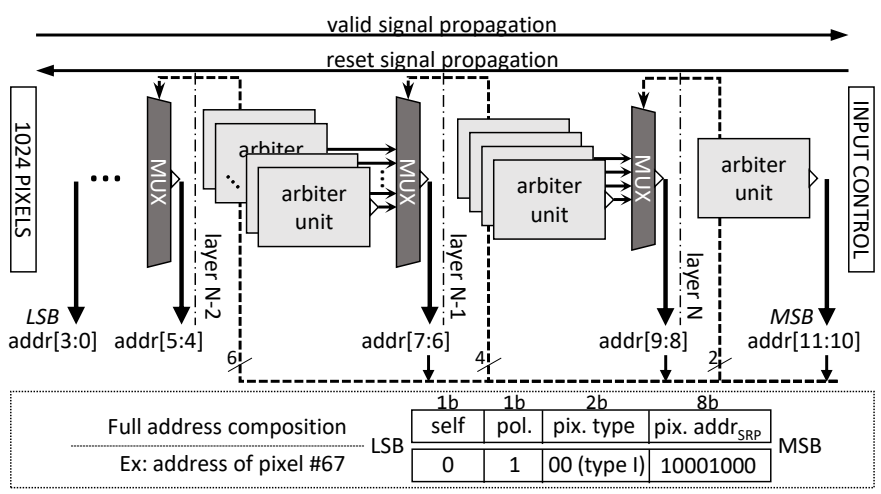

Fig. 5: Schematic of the arbiter working principle.

The $50 \%$ drop in precision when $L_{k}$ decreases from $8 \mathrm{~b}$ to $7 \mathrm{~b}$ led us to set it at $8 \mathrm{~b}$.

3) 3D-Enabled Optimization: To take advantage of the distributed parallel interconnect of the macropixel stack, each core embeds its own readout module to directly access its pixels. We customize the spike addressing which enables us to design a specific network mapping strategy we call mapping by Smallest Repeatable Pattern (SRP). Mapping consists in listing the target neurons and associated weights from the address of an input spike.

A SRP is the smallest block of pixels and RF centers that permits to fully represent the CSNN when meshed uniformly. With $d_{p i x}=2$, the SRP is a group of $2 \times 2$ pixels, as depicted Fig. 4 . In step 1 , the connections between every pixel of a SRP and neighboring neurons are found by taking a window of width $W_{R F}$ around this pixel and looking at the RF centers inside this window. Then in step 2, for each pixel of a SRP, the associated target neurons are mapped by their relative addresses in $\triangle S R P$. Finally in step 3, for each target neuron of each pixel composing a SRP a $12 \mathrm{~b}$ word is stored in the mapping memory, composed of the $\triangle S R P s$ coordinates (both stored on 2 bits) and the eight $1 \mathrm{~b}$ weights $w_{i}$ associated to each kernel potential. Pixels type I, II (a \& b), and III respectively have 9, 6, and 4 target neurons. The full mapping memory thus requires only $(9+$ $6+6+4) \times 12=300 b$.

Using SRP to describes pixels to RF centers connections mapping information is only dictated by the stride $d_{\text {pix }}$. It is independent of the total number of neurons evaluated or of the core position with respect to the matrix of pixels. Hence, no overhead would be induced by tiling cores as illustrated Fig. 1 . 


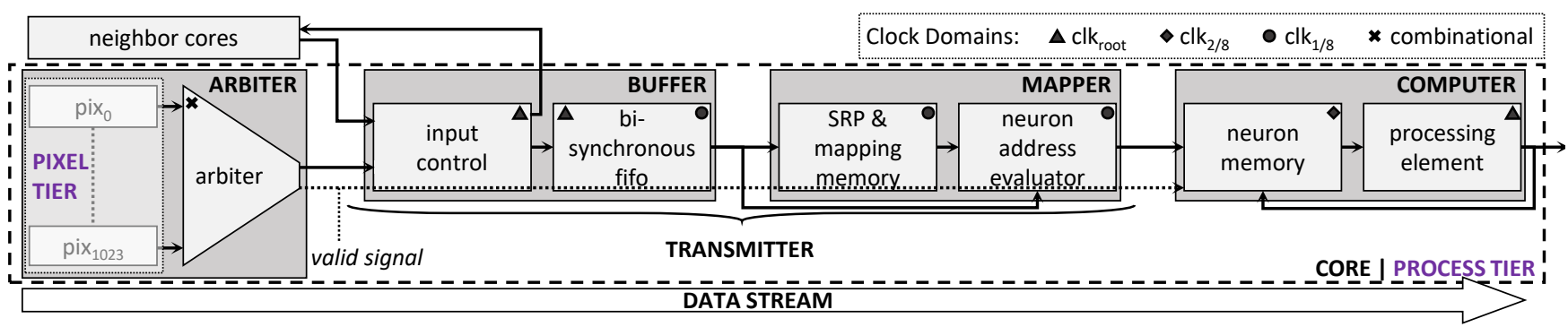

Fig. 6: Overview of the data-stream architecture of the neural processing unit.

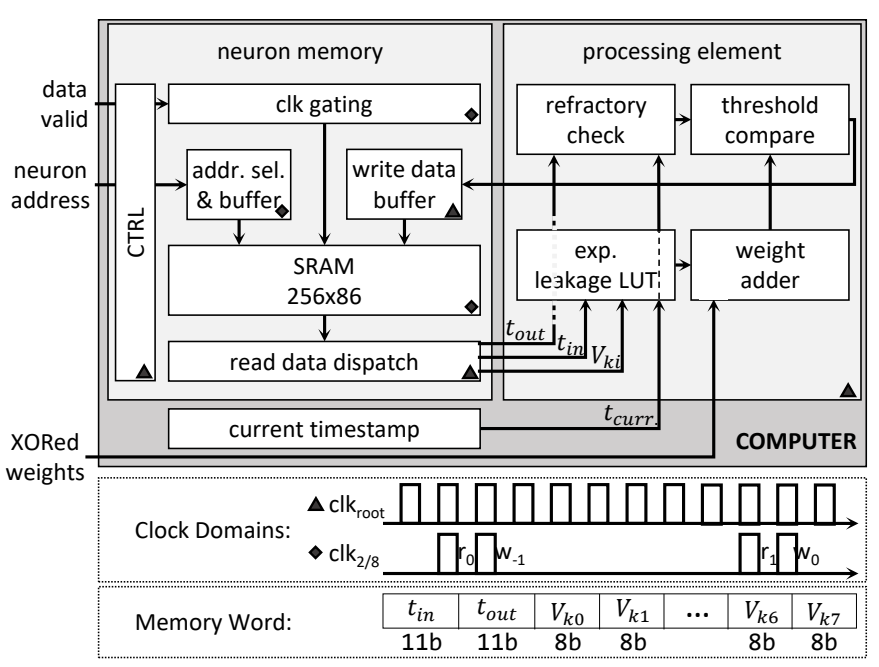

Fig. 7: Schematic of the computer behavior.

\section{Number of Pixels and Target Performances}

For deciding the number of neurons $N_{\text {neuron }}$ a core evaluates, we look at the impact on minimal frequency $f_{\text {root }}$ and core area $A_{M P}$ requirements. $f_{\text {root }}$ is directly proportional to the average pixel input event rate $f_{p i x}$, the number of target neurons per input spike $N_{R F_{\max }}$ - nine for pixel type I -, the number of kernels evaluated per neuron $N_{k}$, and $N_{\text {neuron }}$. The maximal core area $A_{\max }$ is constrained by the number of pixels $N_{p i x}$ of the macropixel stack and their pitch $p_{p i x}$. Also, as a core must instantiate its own SRAM to store neuron states (eight $8 \mathrm{~b}$ kernel potentials and two $11 \mathrm{~b}$ timestamps accounting for $86 \mathrm{~b}$ memory words), the minimal core area is bounded from bellow by the memory cut area $A_{\text {mem }}$.

The core is designed with a single $\mathrm{PE}$, and $f_{p i x}$ and $p_{p i x}$ are taken to compete with the state of the art event-based imager, namely $3.16 \mathrm{kev} / \mathrm{s} /$ pix (maximum internal pixel event rate) and $5 \mu \mathrm{m}$ respectively [7]. With that, the right graph of Fig. 3 depicts the evolution of the area boundaries (green) and $f_{\text {root }}$ (blue) as a function of $N_{\text {pix }}$. When $N_{\text {pix }}<1024, A_{\text {mem }}>A_{\max }$ which is not possible, so $N_{\text {pix }}$ should at least be 1024 . However, with $N_{\text {pix }} \geq 2048, f_{\text {root }}$ is at least $530 \mathrm{MHz}$, which could lead to important power consumption. So, $N_{\text {pix }}$ is set at 1024 , resulting in a macropixel composed of $32 \times 32$ pixels on top of a core evaluating 265 spiking convolutional neurons.

\section{Proposed Architecture}

The overall architecture of our data-stream neural processing unit is illustrated Fig. 6. The behavior is kept non-synchronous with combinatorial circuitry up to the input control module where the signal is sampled by a metastable tolerant synchronizer. After that it is fully synchronous with several clock domains and clock gating levels to reduce power consumption of each module individually.

\section{A. Arbiter}

The spike first passes through the arbiter, where its address is encoded. The arbiter implemented, illustrated Fig. 5 is adapted from [23] with digital electronics and modified address encoding protocol to avoid using tristate buffers, which are replaced by multiplexers.

To emit an event, a pixel sets at 1 its valid signal which then propagates through the arbiter up to the input control module. The input control synchronously samples the valid signal and send a reset pulse. When the rightmost arbiter unit (AU) receives the reset signal, it encodes a $2 \mathrm{~b}$-address that corresponds to its input $\mathrm{AU}$ with active signal high.The multiplexer of layer $\mathrm{N}$ uses this $2 \mathrm{~b}$-address for selecting the next correct $\mathrm{AU}$ to which propagate the reset signal and from whom to read its encoded $2 b$-address.This $2 b$ address is concatenated with the previous one to generate a $4 \mathrm{~b}$-address that will be used by the multiplexer of layer $\mathrm{N}-1$. This goes on sequentially. The SRP address $a d d r_{S R P}$ of the pixel is encoded by concatenation of every $2 b$ address. The AU closest to pixels directly encodes the pixel type. It also conserves the bit of polarity pol. encoded by the pixel and set at 0 a bit called self to indicate that the event does not come from a neighbor MP. These four elements compose the full event address.

\section{B. Transmitter}

The transmitter is composed of a buffer and the pixel to neuron mapper. The buffer includes the input control which manages spikes from the arbiter and neighboring MPs (distinguished with the self bit) and stores them in a bisynchronous FIFO [24]. When an event is available in the FIFO, the mapper fetches it and directly passes the valid signal and the $a d d r_{S R P}$ downstream. The event pixel type dictates which target neurons mapping information $(\triangle S R P s$ and associated weights) to load. The neuron address evaluator first decomposes the $a d d r_{S R P}$ into SRP coordinates $\mathrm{SRP}_{\mathrm{x}}$ and $\mathrm{SRP}_{\mathrm{y}}$. It then recomposes the target neuron address as $a d d r_{R F}=\left[\mathrm{SRP}_{\mathrm{x}}+\right.$ $\left.\Delta S P R_{x} ; \mathrm{SRP}_{\mathrm{y}}+\Delta S R P_{y}\right]$. It also XOR the 8 weights with the event polarity and passes the resulting vector of bits to the computer module along with $a d d r_{R F}$. This operation is repeated at $f_{1 / 8}=1 / 8 \times f_{\text {root }}$ for every target neuron associated with the pixel type of the event (e.g. 9 times for pixel type I).

\section{Computer}

The computer is composed of the neuron state memory that stores the 256 neuron states and a single PE that updates the eight kernel potentials. Fig. 7 depicts the dataflow inside the computing unit of the core.

1) Neuron Memory: $a d d r_{R F}$ is used to load the neuron's previous state at the read cycle $r_{0}$ (see chronograms Fig. 7). A single memory word contains the eight kernel potentials $V_{k_{i}}$ and the timestamps $t_{i n}$ and $t_{\text {out }}$, corresponding to the times of the last input and outputted spikes respectively. The $V_{k_{i}}$ are sequentially sent to the PE for update, one $V_{k_{i}}$ by clock cycle at $c l k_{\text {root }}$. To guarantee functional read/write 

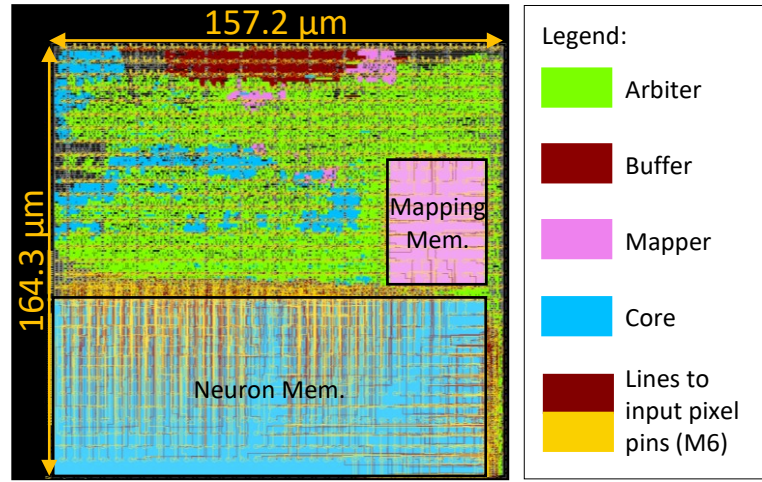

Fig. 8: Layout of a single neural core for $32 \times 32$ pixels.

synchronization with a single port SRAM, a write data buffer is placed at the input of the memory data port. It consists in seven registers in parallel, each sequentially storing an updated $V_{k_{i}}$. The last updated $V_{k_{7}}$ is not stored in a register but directly written, at write cycle $w_{0}$, along with the seven others $V_{k_{i}}$ and the timestamps $t_{\text {in }}$ and $t_{\text {out }}$, at the same $a d d r_{R F}$ which has been memorized by the address selector and buffer. The bits corresponding to $t_{\text {out }}$ are masked, excepted when the neuron fires, in which case all $V_{k_{i}}$ are set to zero at write time. The control module manages clock gating and read and write addresses selection throughout each read/write cycle. The SRAM works under the $c l k_{2 / 8}$ clock domain, and is clock gated if the valid signal propagated all the way from the arbiter is low.

2) Processing Element: The PE is fully combinational. It first applies leakage on $V_{k_{i}}$ - as described sec. III-B2. Then, the XORed weight associated to the $\mathrm{i}^{\text {th }}$ kernel selects if the leaked $V_{k_{i}}$ is added or subtracted one. $V_{k_{i}}$ is now fully updated and compared with $V_{t h}$. If $V_{k_{i}}>V_{t h}$, the neuron spikes. Otherwise the memory simply stores the updated $V_{k_{i}}$ and $t_{i n}$ at $a d d r_{R F}$ - as explained above. In parallel, the refractory checker verifies $t_{\text {curr. }}-t_{\text {out }}<T_{\text {refrac }}$. If the condition is true the neuron is in refractory period, in which case the spiking operation is not allowed, even if $V_{k i}>V_{t h}$ is true. If an output spike is produced, the PE sends an event word composed of $\left[a d d_{S R P}, t_{c u r r .}, i\right]$ to a virtual output port.

\section{Results}

\section{A. Methodology}

We evaluated the area and power consumption of the circuit based on post-layout simulations using uniform random spiking patterns as input to the neural core. All numbers presented below are associated to a single neural core. The power contributions of pixels and 3D interconnects are not considered in this work. Only the neural processing block is designed, and pixel-related input pins are distributed on top the core at metal layer 6 , with pitches of $5 \mu \mathrm{m}$. Nevertheless, the IP proposed here could be straightforwardly tiled and integrated within a full 3D stacked EB imager conception flow using available $3 \mathrm{D}$ process design kits. The snapshot of the layout is shown Fig. 8.

The design was synthesized with Synopsys ${ }^{\odot}$ Design Compiler using ST 28nm FDSOI technologies standard libraries. Place and route was realized with Cadence ${ }^{\Theta}$ Innovus $^{\mathrm{TM}}$. Finally, after signoff timing and power analysis with the Synopsys PrimeTime ${ }^{\odot}$ suite, the core power evaluation is done through post-layout simulations under Mentor $^{\circledR}$ Questa $^{\circledR}$ Simulator.

\section{B. Input Rate, Chip Frequency and Power Consumption}

Two different target $f_{\text {root }}$ for synthesis and simulations are tested: $400 \mathrm{MHz}$ and $12.5 \mathrm{MHz}$. These frequency values permit to easily
TABLE II: Comparison with State of the Art SNN Accelerators

\begin{tabular}{|c|c|c|c|c|c|c|c|}
\hline Reference & \multicolumn{2}{|c|}{ This Work } & {$[18]$} & {$[19]$} & {$[21]$} & \multicolumn{2}{|c|}{$[20]$} \\
\hline Implementation & \multicolumn{2}{|c|}{ Digital } & Digital & Digital & Digital & \multicolumn{2}{|c|}{$\frac{\text { Digital }}{\text { Digal }}$} \\
\hline IC Technology & \multicolumn{2}{|c|}{$28 \mathrm{~nm}$ FDSOI } & $28 \mathrm{~nm}$ FDSOI & $65 \mathrm{~nm}$ & 14nm FinFET & \multicolumn{2}{|c|}{ 10nm FinFET } \\
\hline Data Obtained From & \multicolumn{2}{|c|}{ Post-Layout } & Chip & Chip & Post-Layout & \multirow{2}{*}{\multicolumn{2}{|c|}{ Chip }} \\
\hline \begin{tabular}{|l|} 
NN Type \\
\end{tabular} & \multicolumn{2}{|c|}{ C-SNN } & FC-SNN & FC-BaNN ${ }^{c}$ & Various & Various & \\
\hline Core Area $\left(\mathrm{mm}^{2}\right)$ & \multicolumn{2}{|c|}{0.026} & 0.086 & 10.08 & 0.4 & \multirow{2}{*}{\multicolumn{2}{|c|}{1.72}} \\
\hline Neu. Behaviors (nb) & \multirow{2}{*}{\multicolumn{2}{|c|}{$\frac{1}{256}$}} & 20 & 1 & 6 & & \\
\hline Neu. per Core (nb) & & & 256 & 1194 & $\max .1024$ & \multicolumn{2}{|c|}{$\frac{1}{64}$} \\
\hline Syn. Weights Storage & \multicolumn{2}{|c|}{ 1bit SRAM } & 3+1bit SRAM & SRAM & 1 to 9 bit SRAM & \multicolumn{2}{|c|}{ 7bit SRAM } \\
\hline On-Chip Training & \multirow{2}{*}{\multicolumn{2}{|c|}{$\begin{array}{c}\text { No } \\
30.4 \mathrm{k}\end{array}$}} & Yes & Yes & Yes & \multicolumn{2}{|c|}{ Yes } \\
\hline Synapses per Core (nb) & & & $64 \mathrm{k}$ & $238 \mathrm{k}$ & $1 \mathrm{M}$ to $114 \mathrm{k}$ & \multicolumn{2}{|c|}{$16 \mathrm{k}$} \\
\hline Neu. Density $\left(\mathrm{nb} / \mathrm{mm}^{2}\right)$ & \multicolumn{2}{|c|}{$9.8 \mathrm{k}$} & $3.0 \mathrm{k}$ & $0.1 \mathrm{k}$ & $\max .2 .6 \mathrm{k}$ & \multicolumn{2}{|c|}{$2.4 \mathrm{k}$} \\
\hline Syn. Density $\left(\mathrm{nb} / \mathrm{mm}^{2}\right)$ & \multicolumn{2}{|c|}{$1.17 \mathrm{M}$} & $741 \mathrm{k}$ & $23.7 \mathrm{k}$ & $2.5 \mathrm{M}$ to $285 \mathrm{k}$ & \multicolumn{2}{|c|}{$595 \mathrm{k}$} \\
\hline Supply Voltage (V) & \multicolumn{2}{|c|}{1.2} & $0.55-1.0$ & $0.8-1.2$ & $0.5-1.25$ & \multicolumn{2}{|c|}{$0.45-0.9$} \\
\hline Chip Frequency (MHz) & 400 & 12.5 & 75 & 20 & - & 105 & 506 \\
\hline SOP/s & $194.4 \mathrm{M}$ & $16.7 \mathrm{M}^{*}$ & $37.5 \mathrm{M}$ & - & $\min .285 .7 \mathrm{M}$ & $81.3 \mathrm{M}$ & \begin{tabular}{|l|}
$393.8 \mathrm{M}$ \\
\end{tabular} \\
\hline Energy per SOP & 4.8 & 2.86 & $12.7(0.55 \mathrm{~V})$ & - & $>23.6(0.75 \mathrm{~V})$ & $3.8(0.525 \mathrm{~V})$ & $8.3(0.9 \mathrm{~V})$ \\
\hline Total Core Power $(\mu \mathrm{W})$ & 948.4 & 47.6 & $476.3^{\mathrm{a}}$ & $23.6 \mathrm{k}(0.8 \mathrm{~V})^{6}$ & $6.7 \mathrm{k}$ & $308.75^{\mathrm{a}}$ & $3.3 \mathrm{k}$ \\
\hline
\end{tabular}

${ }^{\mathrm{a}}$ Includes IO and classical to event-based input converter.

${ }^{\mathrm{b}}$ Obtained from SOP/s and energy per second values, normalized by the number of cores ${ }^{\mathrm{c}} \mathrm{BaNN}$ stands for Binarized activation Neural Network.

represent timestamps with LSB corresponding to $25 \mu \mathrm{s}$. They are adapted to manage the $720 \mathrm{p}$ equivalent input event rates of $3.5 \mathrm{Gev} / \mathrm{s}$ and $300 \mathrm{Mev} / \mathrm{s}$ respectively. The former is close to the maximal internal event rate of $2.92 \mathrm{Gev} / \mathrm{s}$ measured by [7], and the latter is the nominal event rate for comparing EB sensors. As our core manages 900 times less pixels than a full 720p sensor, the input event rates of $3.5 \mathrm{Gev} / \mathrm{s}, 300 \mathrm{Mev} / \mathrm{s}$, and $100 \mathrm{kev} / \mathrm{s}$ are scaled down for simulation at $3.89 \mathrm{Mev} / \mathrm{s}, 333 \mathrm{kev} / \mathrm{s}$ and $111 \mathrm{ev} / \mathrm{s}$ respectively.

Fig.9 illustrates the power consumption of a single core obtained from post layout simulations at these different frequencies and event rates. The $400 \mathrm{MHz}$ permits to handle a huge number of events, however, a power consumption approaching $1 \mathrm{~mW}$ is not adapted for an embedded device. Moreover, a CR of 10 still leads to $350 \mathrm{Mev} / \mathrm{s}$ in output, easily corresponding to a few Gbit/s when encoding spikes individually with a neuron address, a timestamp, and a kernel number. Thus, $12.5 \mathrm{MHz}$ is more suited for embedding our core into an actual device, consuming $47.6 \mu \mathrm{W}$ at the nominal input event rate. The SRAMs and most of the registers being clock gated when no data is available, the power consumption drops by $2.5 \mathrm{x}$ to $19 \mu \mathrm{W}$ at the minimal input activity.

\section{Comparison With Other Works}

A common metric to evaluate the efficiency of SNN evaluators is the energy per Synaptic Operation (SOP). A SOP is defined as a complete update operation onto one kernel potential of a neuron. For instance, a spike impinging from a pixel type I updates 9 neurons, each characterized by 8 kernels, thus resulting in 9x $8=72$ SOPs. The four different pixel types characterizing a SRP lead to an average number of target neurons per input spike of $25 / 4=6.25$, thus the number of SOP per second at an input event rate of $333 \mathrm{kev} / \mathrm{s}$ is $6.25 \times 8 \times 333 \mathrm{k}=16.65 \mathrm{M} \mathrm{SOP} / \mathrm{s}$. With that in mind results the reader can find a comparison with other SNN accelerators table II.

On top of that, to characterize power consumption of an eventual 3D stacked EB imager, we extract the dynamic energy per event, as proposed in [7], [10]. Aiming for a scalable metric independent of the matrix pixel size, we divide this metric by the number of pixels, resulting in an energy per event per pixel. At $333 \mathrm{kev} / \mathrm{s}$ and $12.5 \mathrm{MHz}$, our core consumes 93.0aJ/ev/pix. The reader can find an exhaustive comparison with EB imagers in table III.

\section{Discussion}

A strong point in our design resides in having the arbiter local to our core. Arbitrating 1024 pixels with 4-input to 1-output AUs requires only 5 layers. Moreover, with $f_{p i x}=3.16 \mathrm{kHz}$ the average inter-spike delay for 1024 pixels is $309 \mathrm{~ns}$, which corresponds to a minimum sampling frequency of $324 \mathrm{kHz}$. A full $720 \mathrm{p}$ sensor would 
TABLE III: Comparison with State of the Art EB Imagers

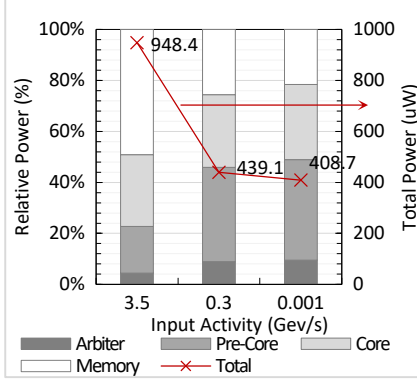

(a) $f_{\text {root }}=400 \mathrm{MHz}$

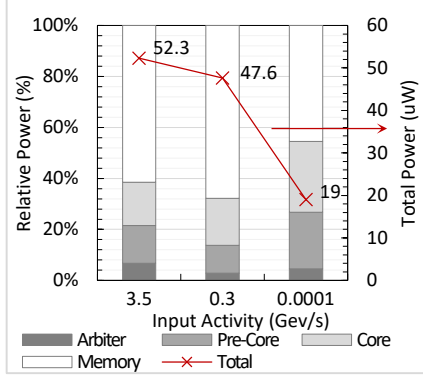

(b) $f_{\text {root }}=12.5 \mathrm{MHz}$
Fig. 9: Post-Layout power distribution for several input event rates. The bars are normalized by the total power (red line).

require 10 arbitration layers and a minimum sampling frequency of $2.92 \mathrm{GHz}$ [7]. This is a strong argument in favor of 3D readout per $\mathrm{MP}$, in addition to requiring only $300 \mathrm{~b}$ of storage for mapping the full network.

We hardwired most of the algorithm parameters, drastically reducing our core flexibility. However, it enables us to make it fit in the $5 \mu \mathrm{m}$ pixel pitch constrained area representing only $0.026 \mathrm{~mm}^{2}$.

Nevertheless, because the floorplan is not $100 \%$ dense, several tracks can be followed to improve our design. For example we could add more programmability to our core or implement 4 PEs in parallel instead of a single one which would permit to reduce $f_{r}$ oot at $3.125 \mathrm{MHz}$.

\section{CONCLUSION}

We see many applications appearing where HDR and HAS imagers are required. EB sensors present both these properties, but come with several drawbacks. To solve their limitations, we deploy a CSNN to filter noise and reduce the output event rate by $10 \mathrm{x}$ while conserving spatial and temporal information. Considering embedded evaluation of the algorithm, we implemented a data stream EB neural core mostly hardwired. By envisaging 3D stacking integration for near-sensor computing directly behind an EB imager, the core area is constrained to $5 \mu \mathrm{m}$-pitch pixels and fits in only $0.026 \mathrm{~mm}^{2}$. It consumes only $2.86 \mathrm{pJ} / \mathrm{SOP}$ at a $720 \mathrm{p}$ equivalent nominal input event rate of $300 \mathrm{Mev} / \mathrm{s}$, corresponding to $93.0 \mathrm{aJ} / \mathrm{ev} / \mathrm{pix}$.

This performance can be achieved thanks to direct access to pixels with 3D IC technology. The core instantiates a direct distributed 1024-input pixel encoder, which enables to store the full mono-layer CSNN in only 300 bits and to drastically reduce the chip frequency. Moreover, it can be tiled without overhead to manage high resolution EB image sensors. As a next step we will integrate the proposed neural processing unit within a $3 \mathrm{D}$ stacked $\mathrm{EB}$ imager design for ego-motion evaluation target application.

\section{REFERENCES}

[1] P. Lichtsteiner et al., "A 128 x $128120 \mathrm{~dB} 15$ us Latency Asynchronous Temporal Contrast Vision Sensor," JSSCC, 2008.

[2] C. Posch et al., "A QVGA 143 dB Dynamic Range Frame-Free PWM Image Sensor With Lossless Pixel-Level Video Compression and TimeDomain CDS," JSSCC, 2011.

[3] G. Gallego et al., "Event-based Vision: A Survey," TPAMI, 2020.

[4] K. Sakuma, 3D Integration in VLSI Circuits, 1st ed. Boca Raton: CRC Press., 2018.

[5] P. Vivet et al., "Advanced 3D Technologies and Architectures for 3D Smart Image Sensors," in DATE, 2019.

[6] D. H. Hubel and T. N. Wiesel, "Receptive fields of single neurones in the cat's striate cortex," J Physiol, 1959.

\begin{tabular}{|c|c|c|c|c|c|c|}
\hline \multirow{2}{*}{\multicolumn{2}{|c|}{\begin{tabular}{|l|} 
Reference \\
IC Technology
\end{tabular}}} & \multicolumn{2}{|c|}{ This Work } & [7] & [10] & [11] \\
\hline & & \multicolumn{3}{|c|}{ 3D } & \multicolumn{2}{|c|}{$2 \mathrm{D}$} \\
\hline \multicolumn{2}{|l|}{ Filter Type } & \multicolumn{2}{|c|}{$\begin{array}{c}\text { Convolutional Spiking } \\
\text { Neurons }\end{array}$} & $\begin{array}{l}\text { Regions of } \\
\text { Interest }\end{array}$ & $\begin{array}{c}\text { Event } \\
\text { Counting }\end{array}$ & None \\
\hline \multicolumn{2}{|c|}{ Technological Node } & \multicolumn{2}{|c|}{$\mathrm{None}^{\mathrm{a}}+28 \mathrm{~nm}$ FDSOI } & $\begin{array}{c}90 \mathrm{~nm} \mathrm{BI} \mathrm{CIS} \mathrm{+} \\
40 \mathrm{~nm} \mathrm{CMOS}\end{array}$ & $\begin{array}{l}65 \mathrm{~nm} \\
\mathrm{CMOS}\end{array}$ & $\begin{array}{c}90 \mathrm{~nm} \mathrm{ClS} \\
\mathrm{BSI}\end{array}$ \\
\hline \multicolumn{2}{|c|}{ Implementation Stage } & \multicolumn{2}{|c|}{ Post-Layout } & \multicolumn{3}{|c|}{ Circuit Chip } \\
\hline \multicolumn{2}{|c|}{ Resolution } & \multicolumn{2}{|c|}{$N^{b} \times(32 \times 32)$} & $1280 \times 720$ & $132 \times 104$ & $640 \times 480$ \\
\hline \multicolumn{2}{|c|}{ Pixel Size $(\mu \mathrm{m} 2)$} & \multicolumn{2}{|c|}{$5,03 \times 4,80$} & $4.86 \times 4.86$ & $10 \times 10$ & $9 \times 9$ \\
\hline \multicolumn{2}{|c|}{ Clk Frequency (MHz) } & 400 & 12.5 & 100 & 50 & 50 \\
\hline \multirow{2}{*}{$\begin{array}{l}\text { Input event } \\
\text { rate full res. }\end{array}$} & Low (kev/s) & 100 & 100 & 100 & 100 & 100 \\
\hline & High (Mev/s) & 3500 & 300 & 300 & 180 & 300 \\
\hline \multirow{2}{*}{$\begin{array}{l}\text { Power full res. } \\
(\mathrm{mW})\end{array}$} & Low IN rate & 367.83 & $17.1^{\mathrm{c}}$ & 32 & 0.25 & 27 \\
\hline & High IN rate & 854.01 & $42.8^{c}$ & 84 & 4.9 & 50 \\
\hline Power 1024 & Low IN rate & 408.7 & 19 & 35.6 & 18.6 & 90.0 \\
\hline pix eq. ${ }^{d}(\mu W)$ & High IN rate & 948.9 & 47.6 & 93.3 & 365.5 & 166.7 \\
\hline \multicolumn{2}{|c|}{ Energy/event/pix $(\mathrm{aJ} / \mathrm{pix})$} & 150.7 & 93.0 & 188.1 & 1882.8 & 249.6 \\
\hline \multicolumn{2}{|c|}{ Static Power (nW/pix) } & 399.1 & 18.5 & 34.7 & 18.0 & 87.9 \\
\hline \multicolumn{2}{|c|}{ Max. IN Event Rate (Mev/s) } & $3500^{f}$ & 300 & $2920^{f}$ & 180 & 300 \\
\hline
\end{tabular}

${ }^{a}$ Pixel tier not implemented.

${ }^{\mathrm{b}}$ Results shown for a block of $32 \times 32$ pixels, designed to be scalable.

${ }^{\mathrm{c}}$ Values for an equivalent resolution of $1280 \times 720$ pixels; i.e. $N=900$.

${ }^{\mathrm{d}}$ Values for an equivalent resolution of $32 \times 32$ pixels.

${ }^{\mathrm{e}}$ Dynamic energy, defined as in [10], divided by the total number of pixels.

${ }^{\mathrm{f}}$ Peak internal activity.

[7] T. Finateu et al., "1280 x 720 Back-Illuminated Stacked Temporal Contrast Event-Based Vision Sensor with $4.86 \mu \mathrm{m}$ Pixels, 1.066GEPS Readout, Programmable Event-Rate Controller and Compressive DataFormatting Pipeline," in ISSCC, 2020.

[8] D. Purves et al., "Vision: the Eye," in Neuroscience. Oxford University Press, 2018.

[9] S.-C. Liu et al., "On-Chip AER Communication Circuits," in EventBased Neuromorphic Systems, John Wiley \& Sons, Ltd, 2014.

[10] L. Chenghan et al., "A 132 by $10410 \mu \mathrm{m}$-Pixel $250 \mu \mathrm{W} 1 \mathrm{kefps}$ Dynamic Vision Sensor with Pixel-Parallel Noise and Spatial Redundancy Suppression," in Symposium on VLSI Circuits, 2019.

[11] B. Son et al., "4.1 A 640×480 dynamic vision sensor with a $9 \mu \mathrm{m}$ pixel and 300Meps address-event representation," in ISSCC, 2017.

[12] A. Nose et al., "Design and Performance of a $1 \mathrm{~ms}$ High-Speed Vision Chip with 3D-Stacked 140 GOPS Column-Parallel PEs," Sensors, 2018.

[13] https://www.sony-semicon.co.jp/e/technology/imaging-sensing/.

[14] L. Millet et al., "A 5500-frames/s 85-GOPS/W 3-D Stacked BSI Vision Chip Based on Parallel In-Focal-Plane Acquisition and Processing," JSSCC, 2019.

[15] S. R. Kheradpisheh et al., "STDP-based spiking deep convolutional neural networks for object recognition," Neural Networks, 2018.

[16] F. Paredes-Vallés et al., "Unsupervised Learning of a Hierarchical Spiking Neural Network for Optical Flow Estimation: From Events to Global Motion Perception," IEEE Trans. Pattern Anal. Mach. Intell., 2020.

[17] M. Bouvier et al., "Spiking Neural Networks Hardware Implementations and Challenges: A Survey," ACM JETC, 2019.

[18] C. Frenkel et al., "A 0.086-mm² 12.7-pJ/SOP 64k-Synapse 256-Neuron Online-Learning Digital Spiking Neuromorphic Processor in 28-nm CMOS," IEEE Trans. Biomed. Circuits and Systems, 2019.

[19] J. Park et al., "A 65-nm Neuromorphic Image Classification Processor With Energy-Efficient Training Through Direct Spike-Only Feedback," JSSCC, 2020.

[20] G. K. Chen et al., "A 4096-Neuron 1M-Synapse 3.8-pJ/SOP Spiking Neural Network With On-Chip STDP Learning and Sparse Weights in 10-nm FinFET CMOS," JSSCC, 2019.

[21] M. Davies et al., "Loihi: A Neuromorphic Manycore Processor with On-Chip Learning," IEEE Micro, 2018.

[22] E. Mueggler et al., "The Event-Camera Dataset and Simulator: Eventbased Data for Pose Estimation, Visual Odometry, and SLAM," 2016.

[23] P. Yang et al., "Low-power priority Address-Encoder and Reset-Decoder data-driven readout for Monolithic Active Pixel Sensors for tracker system," NIMPR, Section A, 2015.

[24] I. Miro Panades et al., "Bi-Synchronous FIFO for Synchronous Circuit Communication Well Suited for Network-on-Chip in GALS Architectures," in NOCS'07, 2007. 